\title{
System identification on the seismic behavior of an isolated bridge
}

\author{
Zheng-Kuan Lee ${ }^{1}$, Tzu-Hsiu $\mathrm{Wu}^{2}$ and Chin-Hsiung Loh $^{1, *, \dagger}$ \\ ${ }^{1}$ National Center for Research on Earthquake Engineering, National Taiwan University, Hsinhai Road, \\ Taipei, Taiwan \\ ${ }^{2}$ National Science and Technology Program for Hazards Mitigation, National Taiwan University, \\ Taipei, Taiwan
}

\begin{abstract}
SUMMARY
Seismic isolation devices and technology have been developed in the last two decades and the first seismic-isolated bridge, Bai-Ho Bridge, in Taiwan was completed in 1999. This bridge was equipped with a seismic monitoring system under the Taiwan Strong Motion Instrumentation Program (TSMIP). On 22 October 1999, a moderate earthquake took place and struck the bridge. The seismic monitoring sensors were normally triggered and produced an intact time history for the health monitoring of the bridge system. The data set provided valuable records about the seismic response of the isolation bridge structure. This paper uses this data to explore the vibration mechanism of Bai-Ho Bridge. The analysis includes (1) the global dynamic behavior identification and (2) the local component mechanism of the bridge such as the LRB and boundary condition between the deck and the abutments. Both the EMD + HHT method and the non-linear parametric model were used to identify the model of the bridge structure. Copyright (C) 2003 John Wiley \& Sons, Ltd.
\end{abstract}

KEY WORDS: system identification; lead rubber bearing (LRB); slip-lock model; non-linear dynamic analysis

\section{INTRODUCTION}

Earthquakes pose a threat to bridge structures. Strong ground shaking usually causes damage to bridges and brings significant inconvenience and economic loss to the surrounding communities. Recently there has been activity amongst engineers directed towards gaining further insight into the behavior of bridges using system identification techniques. These techniques involve a process of pattern recognition in the qualitative or quantitative analysis of a specified system. More strictly speaking, these techniques should improve the mathematical model of the

\footnotetext{
* Correspondence to: Chin-Hsiung Loh, National Center for Research on Earthquake Engineering, National Taiwan University, Hsinhai Road, Taipei, Taiwan.

† E-mail: lohc@ncree.gov.tw

Contract/grant sponsor: National Science Council; contract/grant number: NSC90-2711-3-319-200-16.
} 
system by observing and using the system's input/output information. From the identification result, the engineers can then quantify the performance and the serviceability of the bridges.

In the past two decades, there have been many papers regarding the system identification and damage detection of bridge structures. Douglas and Reid [1] applied the pullback and quick release method to excite Rose Creek Bridge. It was the first full-scale bridge experiment subjected to artificial disturbance. Werner et al. [2] studied the signal data of the Meloland Road Overcrossing Bridge under the 1979 Imperial Valley Earthquake. This might be the first bridge recorded with seismic response. In Werner's paper, the mode shapes, frequencies and modal damping ratios were identified. Abdel-Ghaffar and Scanlan [3] conducted an extensive experimental investigation on the Golden Gate Suspension Bridge in San Francisco. They analyzed the dynamic parameters of interest for both wind and earthquake engineering problems. Hoshiya and Maruyama [4] suggested monitoring the vibration signal caused by moving vehicles. Moving vehicles can serve as the source of disturbance. Hogue et al. [5] used free-fall objects upon the deck as the impact force. Loh and Lee [6] used the idea of a substructure system to identify the dynamic properties of structural members by using the seismic data of the New-Lian River Bridge in Taiwan. This paper downgrades the global behavior analysis (modal level) into the local behavior analysis (member level). Lee and Loh [7] used the EMD+HHT methods [8] (a time and frequency space analysis method) to study the vibration signal of vehicle-induced bridge response. Both the dynamic properties of the deck and the truck were identified.

The purpose of this paper is to study the seismic behavior of an isolated bridge. The seismic response data is used to develop a non-linear parametric model of an isolated bridge. As well as the global dynamic behavior of the bridge system, the local mechanisms, such as the isolation device and the boundary condition of abutment, are studied.

\section{PROFILE OF BAI-HO BRIDGE}

Bai-Ho Bridge is located in Tainan County, in the south-western part of Taiwan Island. It is the first seismic isolation bridge in Taiwan. The bridge is composed of three spans, looking like a $\pi$-type bridge. Its deck is a non-prismatic, single-cell, pre-stressed concrete box-girder with stretching spans of $40 \mathrm{~m}, 65 \mathrm{~m}$ and $40 \mathrm{~m}$, as shown in Figure 1. Its piers are made of wall-type columns about $7.6 \mathrm{~m}$ in height and with $1.6 \mathrm{~m} \times 6.5 \mathrm{~m}$ uniform section. Above each pier, a pair of lead rubber bearings (LRB) of rectangular shape $1.52 \mathrm{~m} \times 1.25 \mathrm{~m}$ and height $0.25 \mathrm{~m}$ are installed and connected to the deck. At the abutments, PTFE rubber bearings undertake the vertical load of the deck and serve as a roller mechanism in the longitudinal direction.

Under the Taiwan Strong Motion Instrumentation Program (TSMIP), a seismic monitoring system has been implemented in this bridge. Twenty-four accelerometers have been labeled and distributed along the deck, the piers, and the nearby free-field locations, constructing an intact observing network, as shown in Figure 1. The observation system began to operate in October 1999 and on 22 October 1999, an earthquake with local magnitude 6.4 and focal depth 12.1 kilometres occurred. Its epicenter was located at $23.51^{\circ} \mathrm{N}$ and $120.40^{\circ} \mathrm{E}$, just about 25 kilometres away from Bai-Ho Bridge. All the monitoring sensors installed for the bridge were normally triggered for this event. The recorded maximum peak acceleration was 180 gal at Channel 2 for the nearby free field and 537 gal at Channel 23, located at the juncture of 


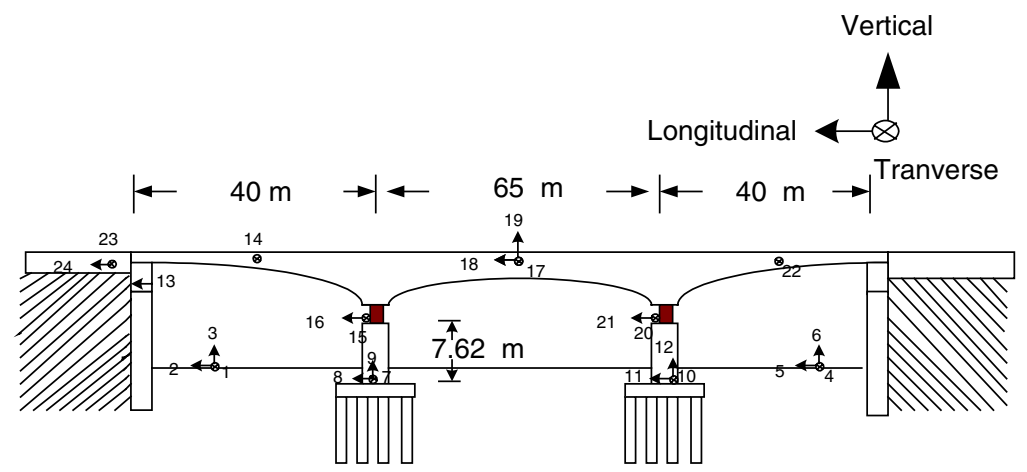

Figure 1. The configuration and monitoring system of Bai-Ho Bridge.
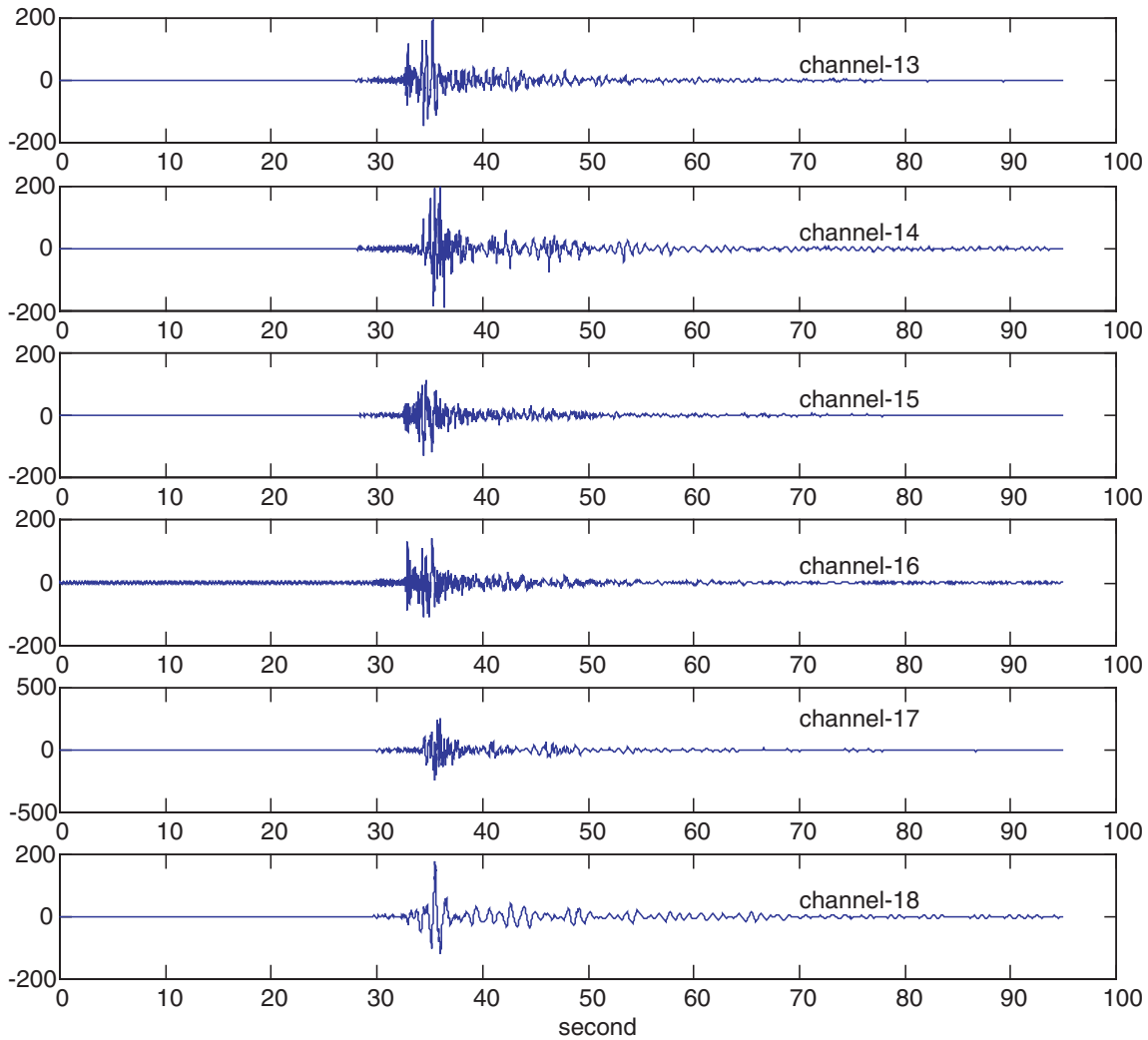

Figure 2. Acceleration time histories for Channel 13 to Channel 18 (units: second and gal).

the abutment and deck in transverse vibration. Figure 2 shows some of the recorded time histories from the seismic monitoring system, and Table I lists the peak acceleration for each channel. 
Table I. Peak acceleration list for all 24 channels.

\begin{tabular}{lccccccc}
\hline $\begin{array}{l}\text { Channel } \\
\text { no. }\end{array}$ & $\begin{array}{c}\text { Peak acc. } \\
\text { (gal) }\end{array}$ & $\begin{array}{c}\text { Channel } \\
\text { no. }\end{array}$ & $\begin{array}{c}\text { Peak acc. } \\
\text { (gal) }\end{array}$ & $\begin{array}{c}\text { Channel } \\
\text { no. }\end{array}$ & $\begin{array}{c}\text { Peak acc. } \\
\text { (gal) }\end{array}$ & $\begin{array}{c}\text { Channel } \\
\text { no. }\end{array}$ & $\begin{array}{c}\text { Peak acc. } \\
\text { (gal) }\end{array}$ \\
\hline 1 & 175 & 7 & 109 & 13 & 198 & 19 & 114 \\
2 & 180 & 8 & 153 & 14 & 201 & 20 & 109 \\
3 & 52 & 9 & 45 & 15 & 130 & 21 & 151 \\
4 & 142 & 10 & 104 & 16 & 142 & 22 & 363 \\
5 & 163 & 11 & 137 & 17 & 261 & 23 & 537 \\
6 & 53 & 12 & 33 & 18 & 180 & 24 & 213 \\
\hline
\end{tabular}

\section{IDENTIFICATION OF GLOBAL DYNAMIC BEHAVIOR}

A new approach to detect the non-linear and non-stationary response signal was introduced by Huang et al. [8] in 1998. In this method two steps were adapted to analyze the data. The first step was to decompose the data into a number of intrinsic mode function (IMF) components by using the empirical mode decomposition (EMD) method. The second step was to apply the Hilbert transform to the IMF components and construct the time-frequencyamplitude spectrum. The dynamic characteristic of structural response can be observed through the spectrum. Based on the above approach the recorded signals from Channels 16, 18, 23 and 24 were analyzed and the spectra are shown in Figure 3. From this figure it is found that the spectrum of Channel 23 (transverse direction) is much more complicated than that of Channel 24 (longitudinal direction). Contact impact might occur at the abutment in the transverse direction. Also, from the spectra of Channel 16 and Channel 18, it is found that the isolation system at the top of the pier reduced the high frequency signals in the longitudinal direction.

In addition to the time-frequency-space analysis, the system realization using input/output information was also applied to identify the fundamental vibration mode of the bridge. The signal at the pier foundation was regarded as the input signal while those on the deck as the output signals. Then the recursive least square method (RLS-method [9]) with a forgetting factor was implemented to identify the vibration characteristic. The result indicates the first mode natural frequency and damping ratio of the bridge system in the longitudinal direction are $1.01 \mathrm{~Hz}$ and $19.35 \%$, respectively. In the transverse direction, however, the dynamic characteristics of the structure system were difficult to identify because of the high frequency signals in the response histories [10].

\section{IDENTIFICATION OF LOCAL STRUCTURE BEHAVIORS}

The seismic response data of Bai-Ho Bridge will be used to identify the local structure behaviors in the longitudinal and transverse directions. The mechanism of the LRB and the boundary conditions at the abutments are regarded as unknown and need to be investigated from the seismic response data. In this analysis, the deck and piers will be assumed to maintain the elastic properties as defined in the design blueprint. 

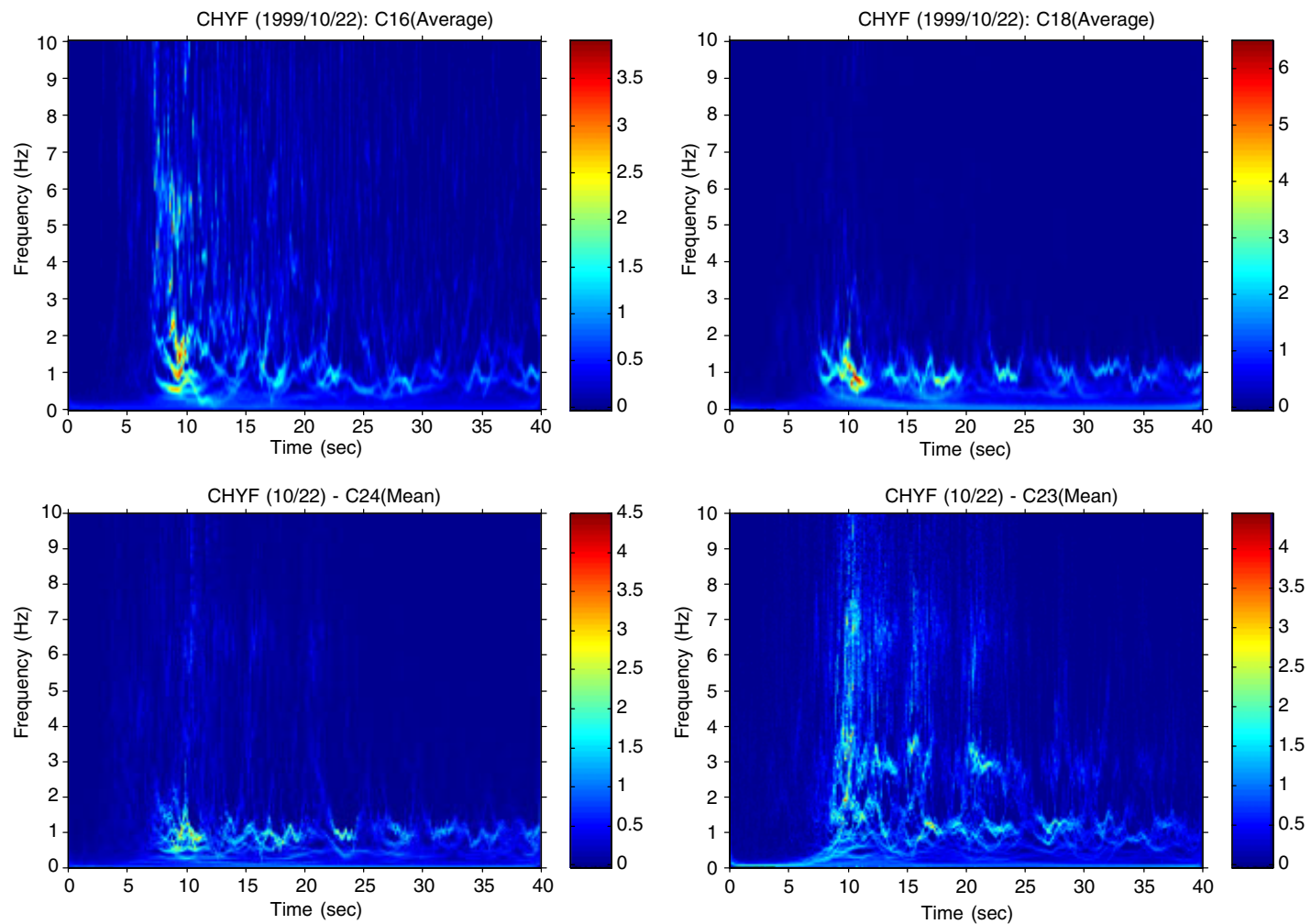

Figure 3. Hilbert spectrum at Channel 16, Channel 18 (top row) and Channel 24, Channel 23 (bottom row).

\section{Identification of the LRB's properties}

Generally speaking, the axial stiffness of the deck is much larger than the shear stiffness of the LRB. From this viewpoint, the deck could be regarded, for simplicity, as a rigid body. As indicated in Figure 4, the inertial force of the deck is transmitted from the isolators. Putting the deforming quantity of the isolator on the abscissa and the inertial force of the deck on the vertical coordinate, one may draw the hysteretic loop for the isolator, as shown in Figure 5. In order to mimic the loop, the Ramber-Osgood model plus a dashpot are adopted, as illustrated in Figure 6, and are expressed in the following two equations:

$$
\begin{aligned}
& E \varepsilon=\sigma+\alpha\left(\frac{|\sigma|}{\sigma_{0}}\right)^{n-1} \sigma \\
& f_{b}=c_{b} \times V
\end{aligned}
$$

where $E$ is the Young's modulus, $\sigma_{0}, \alpha$ and $n$ represent the model parameters, $C_{b}$ is the coefficient of the linear damper and $V$ is the deforming velocity of the LRB. In this model 


\section{Longitudinal Direction}

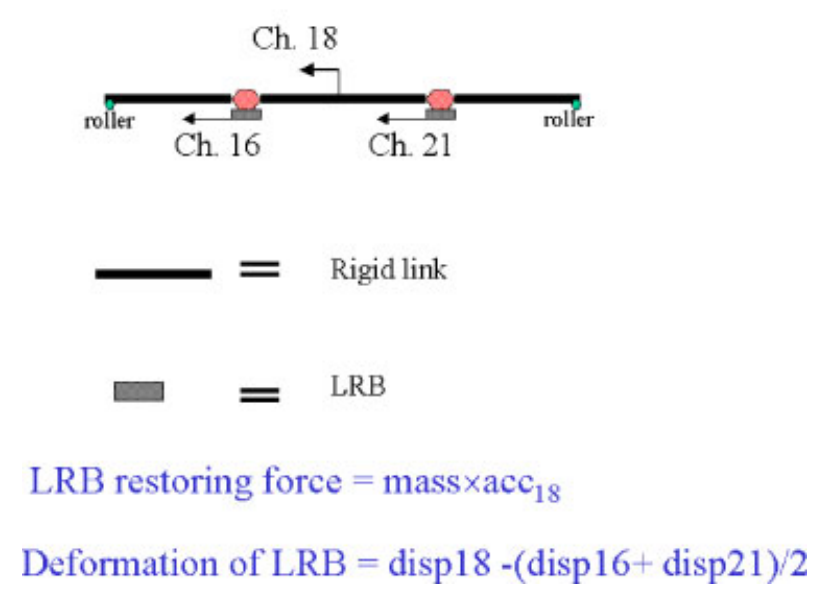

Figure 4. Relationship between the recorded data with the inertial-force of the deck and the deformation of the LRB.

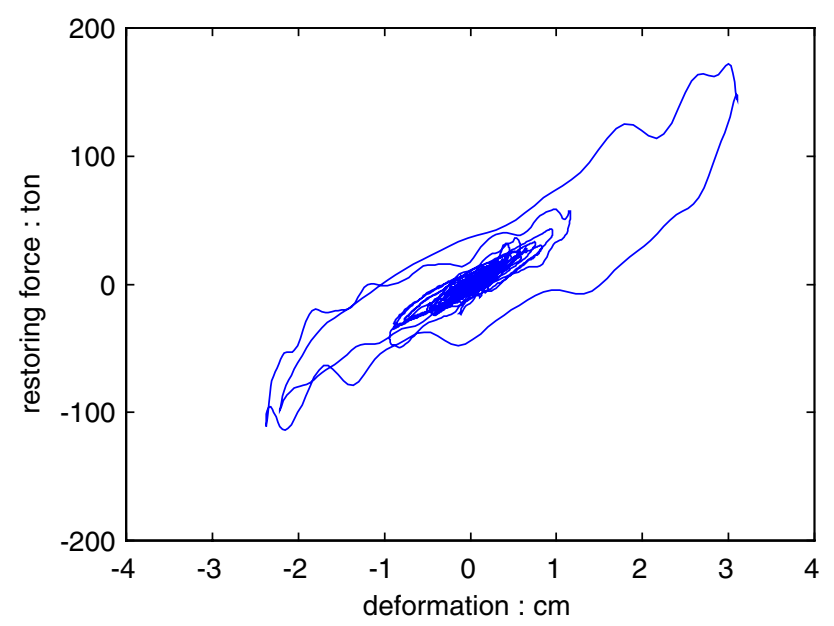

Figure 5. LRB hysteretic loop.

there are five parameters to be determined $\left(E, \sigma_{0}, \alpha, n, C_{b}\right)$. Table II lists the identified optimal model parameters. In agreement with Figure 5, Figure 7 shows the hysteretic behavior of the model with the identified parameters.

From the Fourier spectrum of the seismic response data at Channel 18, as shown in Figure 8 , two obvious peaks are observed $\left(f_{1}=1.08 \mathrm{~Hz}\right.$ and $\left.f_{2}=11.99 \mathrm{~Hz}\right)$. They imply that the vibration mode of the isolated bridge system in the longitudinal direction from this earthquake response data is associated with two mode shapes: the two piers move in the 


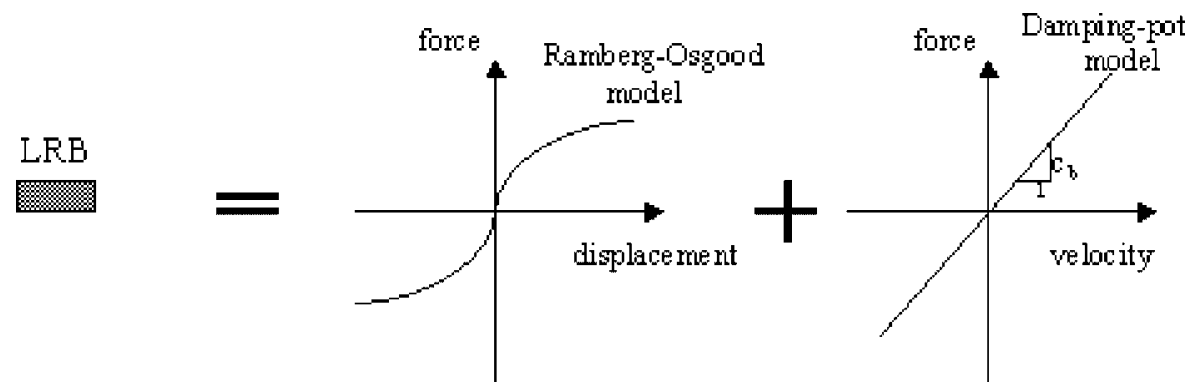

Figure 6. Proposed mathematical model for LRB.

Table II. Optimal Ramber-Osgood model parameters for LRB.

\begin{tabular}{lcccc}
\hline$E$ & $\sigma_{0}$ & $\alpha$ & $N$ & $C_{b}$ \\
\hline $102800 \mathrm{kN} / \mathrm{m}^{2}$ & $600 \mathrm{kN} / \mathrm{m}^{2}$ & 0.15 & 2.5 & $4000 \mathrm{kN}-\mathrm{sec} / \mathrm{m}$ \\
\hline
\end{tabular}

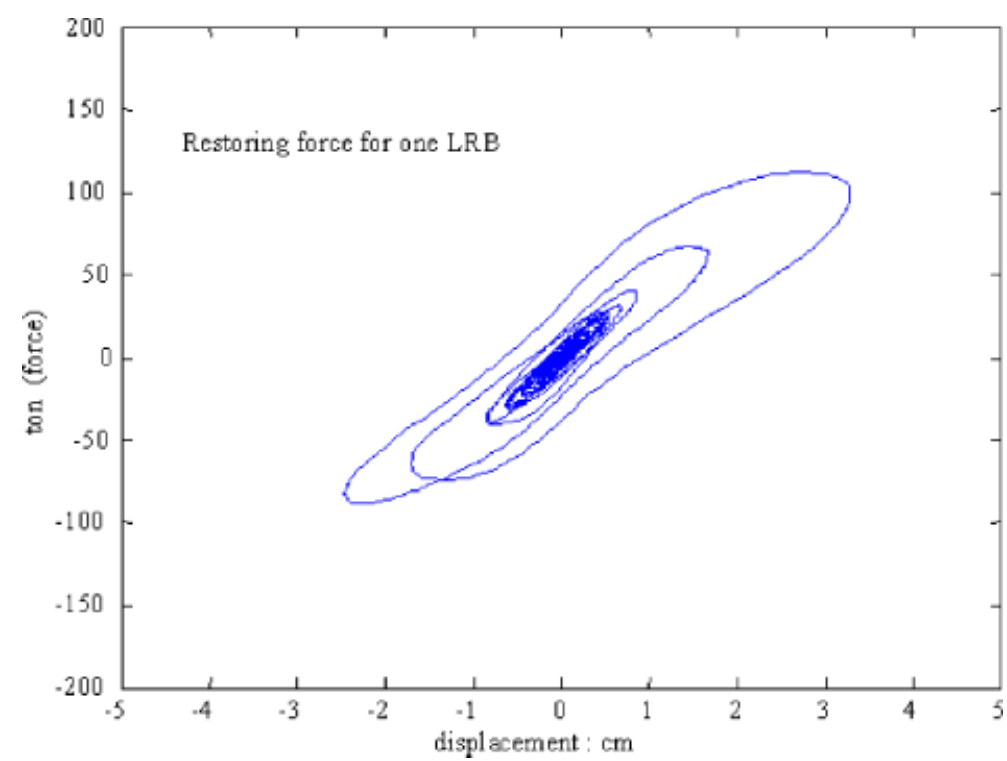

Figure 7. Reconstruction hysteretic loop for LRB (using Ramber-Osgood model with the identified parameters).

same direction for one mode shape and move in opposite directions for the other, as shown in Figure 9. Based on this viewpoint, a modified model in the longitudinal direction with unknown axial stiffness for the bridge system is developed, as shown in Figure 10. In this figure, Channel 8 and Channel 11 are regarded as inputs while Channel 18 is the output. $C_{a}$ is assumed to be a zero damping for the roller mechanism and $k_{a}$ (the stiffness of the deck) is the only unknown parameter to be determined. The pier model is assumed to be the one defined in 


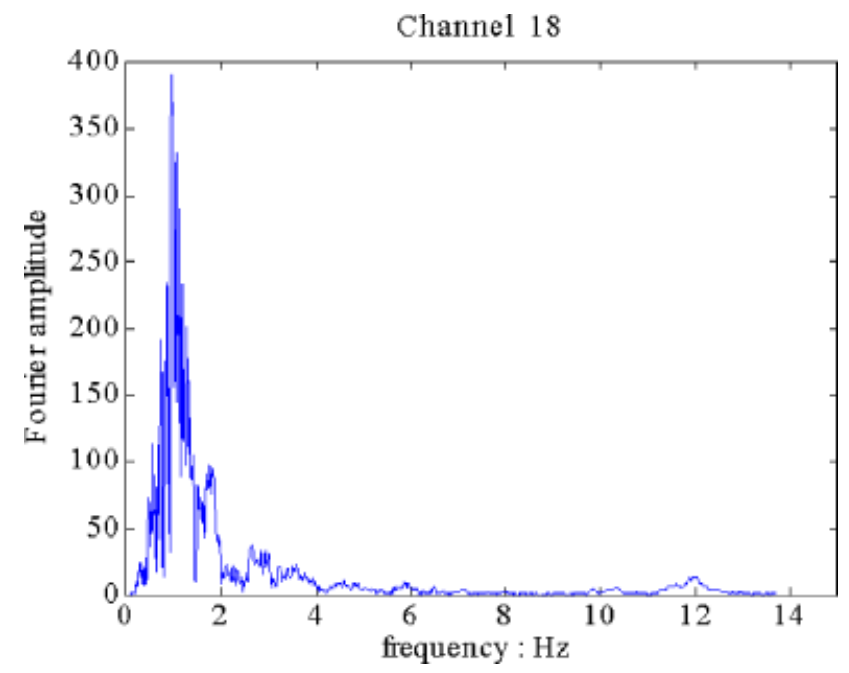

Figure 8. Fourier spectrum for Channel 18, at the center of the mid-span and in the longitudinal direction.

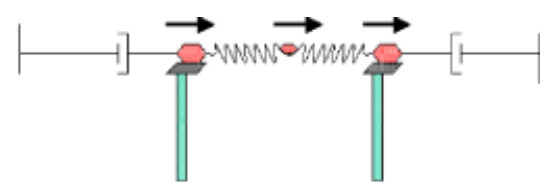

Mode 1: $\mathrm{f}=1.083 \mathrm{~Hz}$

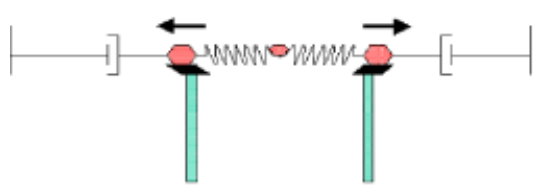

Mode 2: $\mathrm{f}=11.99 \mathrm{~Hz}$

Figure 9. Implied model with two mode shapes and dominant frequencies.

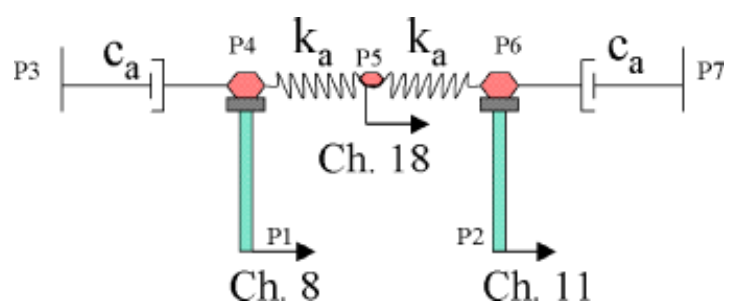

Figure 10. A model with axial stiffness for the deck ( $k_{a}$ for the unknown axial stiffness to be determined). 

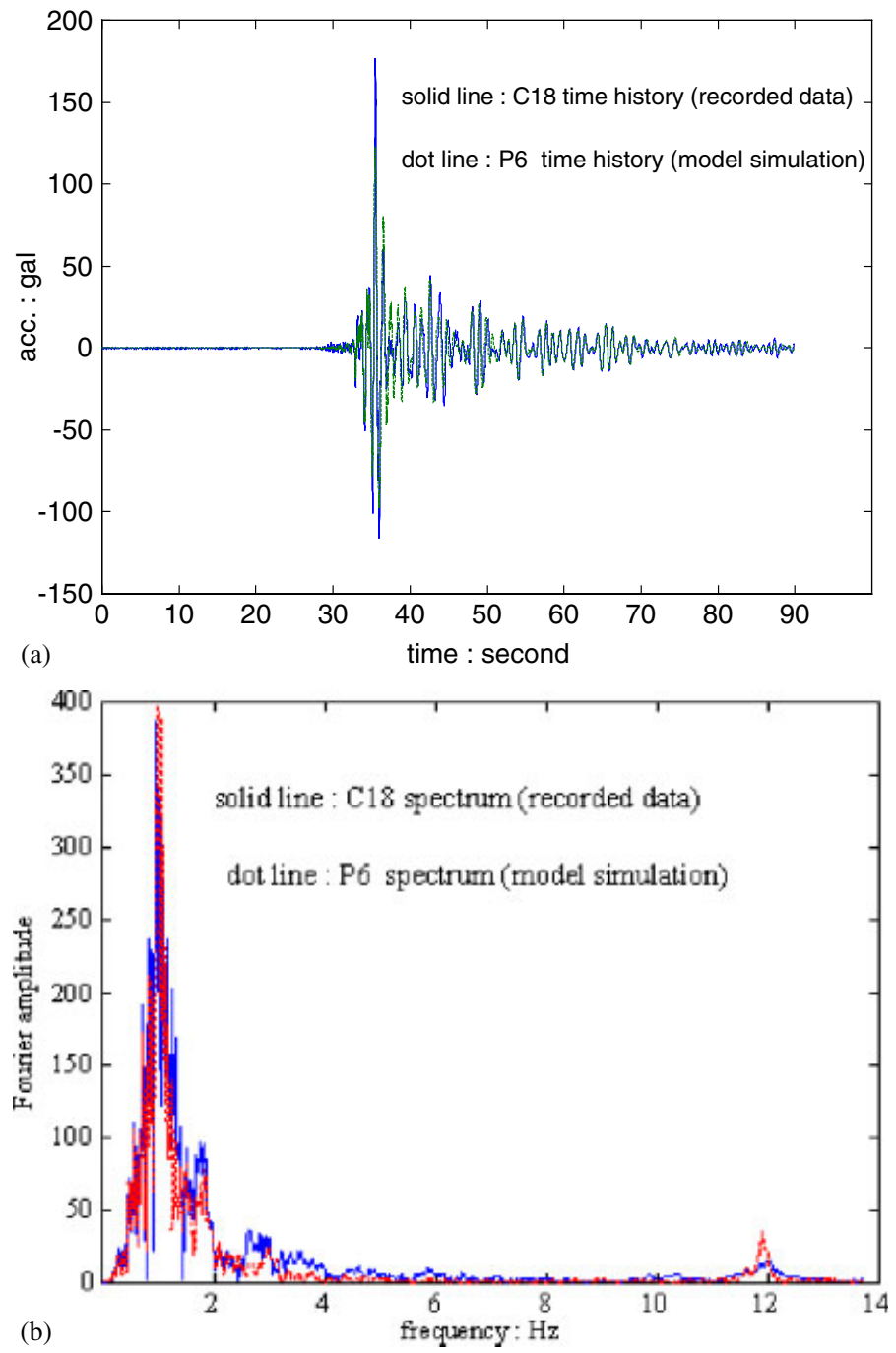

Figure 11. (a) Comparison on acceleration time history between the recorded data and model simulation at Channel 18. (b) Comparison on Fourier spectrum between the recorded data and model simulation at Channel 18 .

the blueprint, and the LRB model to be the one previously identified. By means of trial-anderror to meet the two frequencies, the best parameter for $k_{a}$ is $2.84 E 8 \mathrm{kN} / \mathrm{m}$. In both the time domain and frequency domain, Figure 11 shows the comparison for the response of Channel 18 and thereof the Fourier spectrum between the recorded data and the predicted value.

\section{Identification of the boundary condition of abutments}

One of the main uncertainties in designing a bridge is the boundary condition of the abutments in the transverse direction. It is well known that the abutment's stiffness is not infinite; 


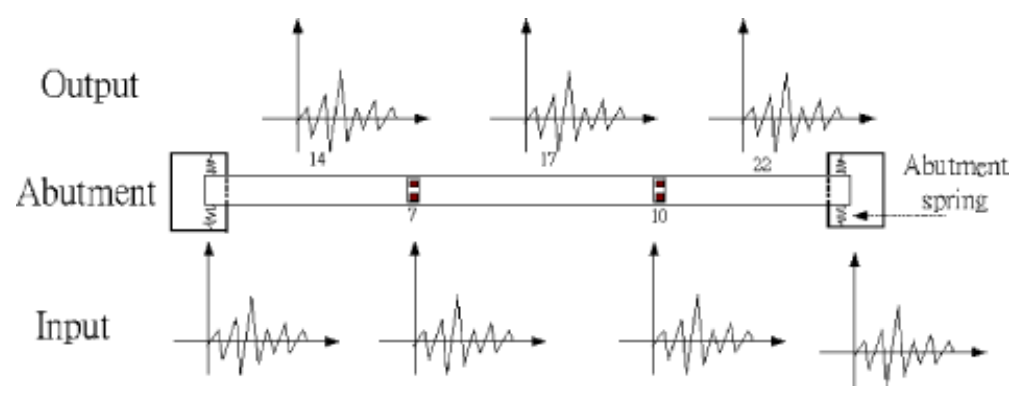

Figure 12. Top view of the bridge with the specified system's output and input.

however, the 'hinge end' model is often applied for convenience and simplicity in the development of the analytical model. Discussion on the boundary condition problem will be made for the bridge system from the seismic response data.

For the transverse vibrations, as shown in Figure 12, the whole structure is specified as a system with input signals at the piers and abutments, and with output signals at the centers of the middle spans. From the signal of Channel 17, two time windows were selected: one is the intense stage (34-38.8 seconds) and the other is the retard stage (50-90 seconds), as shown in Figure 13(a). The Fourier spectra from Channel 14, Channel 17 and Channel 22 in the two different time stages are plotted in Figure 13(b). The identified dominant frequencies are $1.45 \mathrm{~Hz}, 3.33 \mathrm{~Hz}$ and $12.2 \mathrm{~Hz}$ from the intense stage, and $1.22 \mathrm{~Hz}, 2.85 \mathrm{~Hz}$ and $12.0 \mathrm{~Hz}$ from the retard stage. It is found that the dominant frequencies in the intense stage are higher than the corresponding frequencies in the retard stage. Detailed explanation of this observation will be discussed in the following.

Assume the LRB mechanisms in the transverse direction are the same as those in the longitudinal direction (but mutually independent). Two different types of boundary conditions at the juncture of the deck and abutments are considered in FEM analysis. One is the 'free end' and the other is the 'hinge end'. Figure 14(a) and (b) show the mode shapes with the two different boundary conditions. From the comparison in Figure 15, it is found that the natural frequencies of the model with the 'free end' boundary condition are almost identical to those dominant frequencies in the retard stage of the seismic response. On the other hand, the natural frequencies of the model with the 'hinge end' boundary condition are larger than those dominant frequencies in the intense stage of the seismic response. From these observations and discussions, the following viewpoints are drawn:

1. The natural frequencies of the FEM model with the 'free end' boundary condition are close to the dominant frequencies identified from the retard stage. It means that the physical boundary condition for the bridge in the retard stage is similar to the 'free end' mechanism.

2. The identified dominant frequencies from the intense stage are proved to be higher than those in the retard stage. It means that extra stiffness from the boundary between deck and abutment is provided during the intense stage.

3. The natural frequencies for the FEM model with the 'hinge end' are higher than those in the intense stage. It means that the 'hinge end' is too stiff for the physical boundary condition in the intense stage. In other words, the stiffness of the abutments should be finite. 


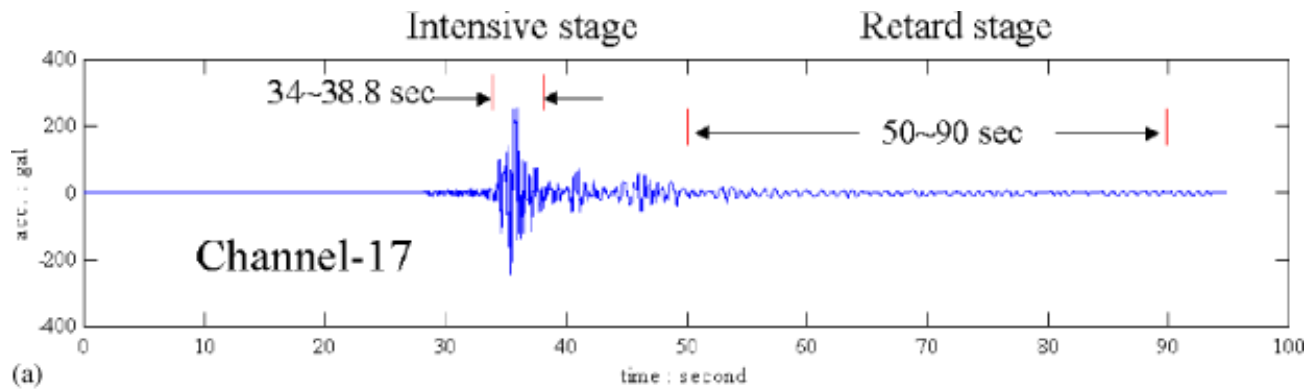

(a)
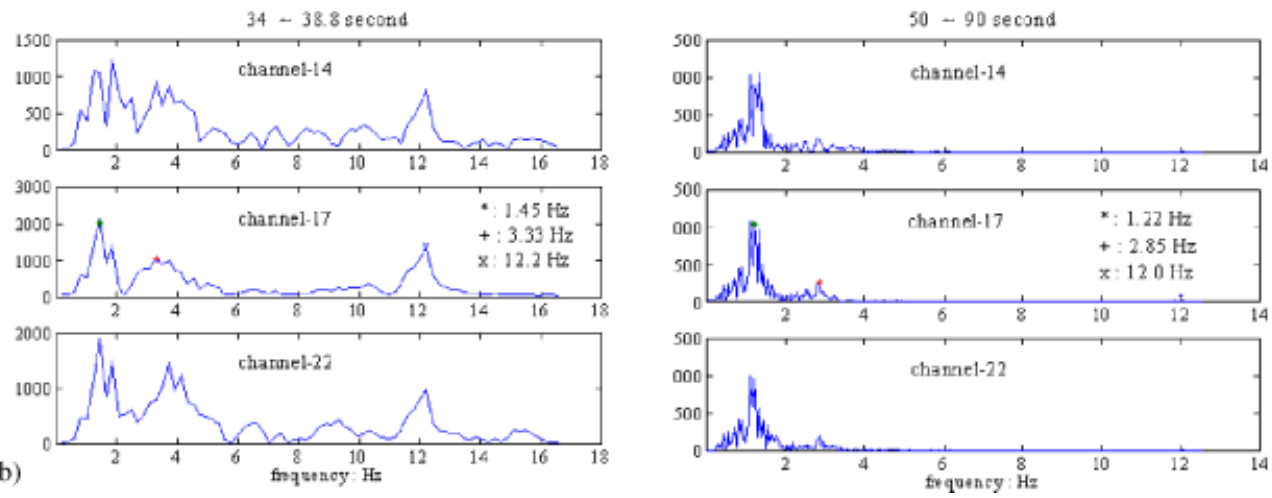

Figure 13. (a) Time history of Channel 17 (Intense and retard stages are specified); (b) Fourier spectrum for Channel 14, Channel 17 and Channel 22 from intense stage (left) and retard stage (right).

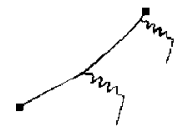

$\mathrm{f}_{1}=1.25 \mathrm{~Hz}$

(a)

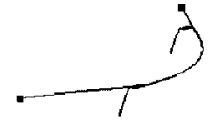

$\mathrm{f}_{2}=2.66 \mathrm{~Hz}$

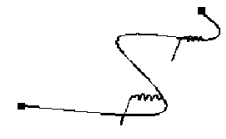

$\mathrm{f}_{3}=11.47 \mathrm{~Hz}$
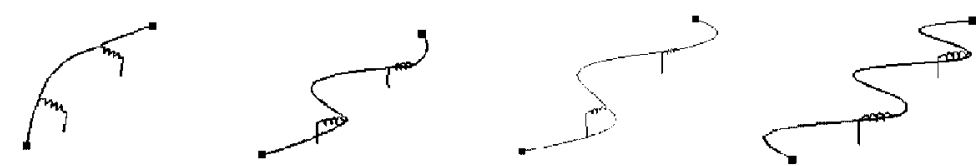

$f_{1}-1.63 \mathrm{~Hz}$

$f_{2}-7.44 \mathrm{~Hz}$

$f_{3}=8.76 \mathrm{~Hz}$

$\mathrm{f}_{4}=21.0 \mathrm{~Hz}$

(b)

Figure 14. Mode shapes in the transverse direction with natural frequencies using (a) 'free end' boundary condition, and (b) 'hinge end' boundary condition. 


\section{Model Analysis}
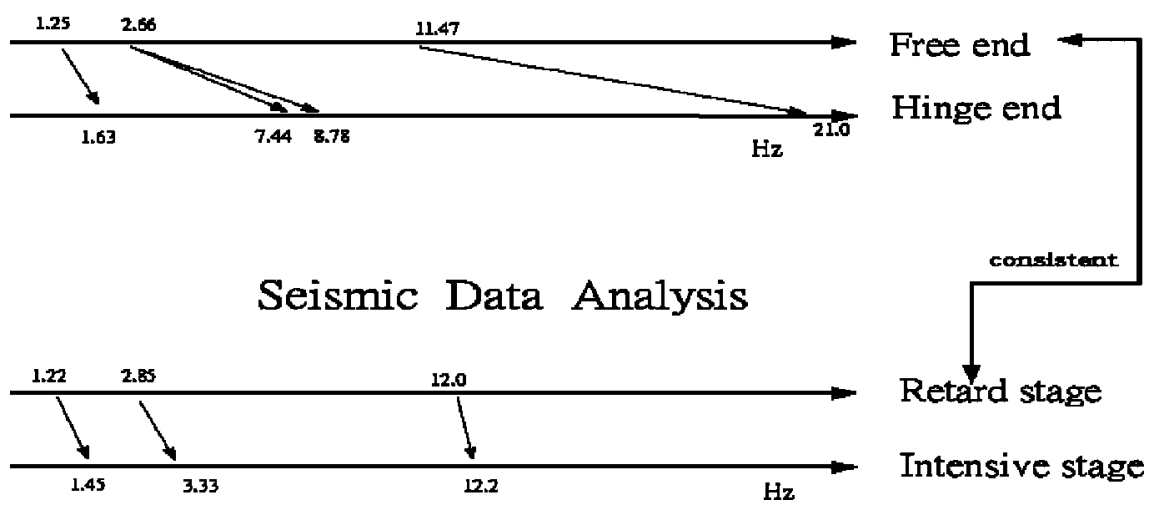

Figure 15. Characteristic frequency map of model analysis and seismic data analysis.
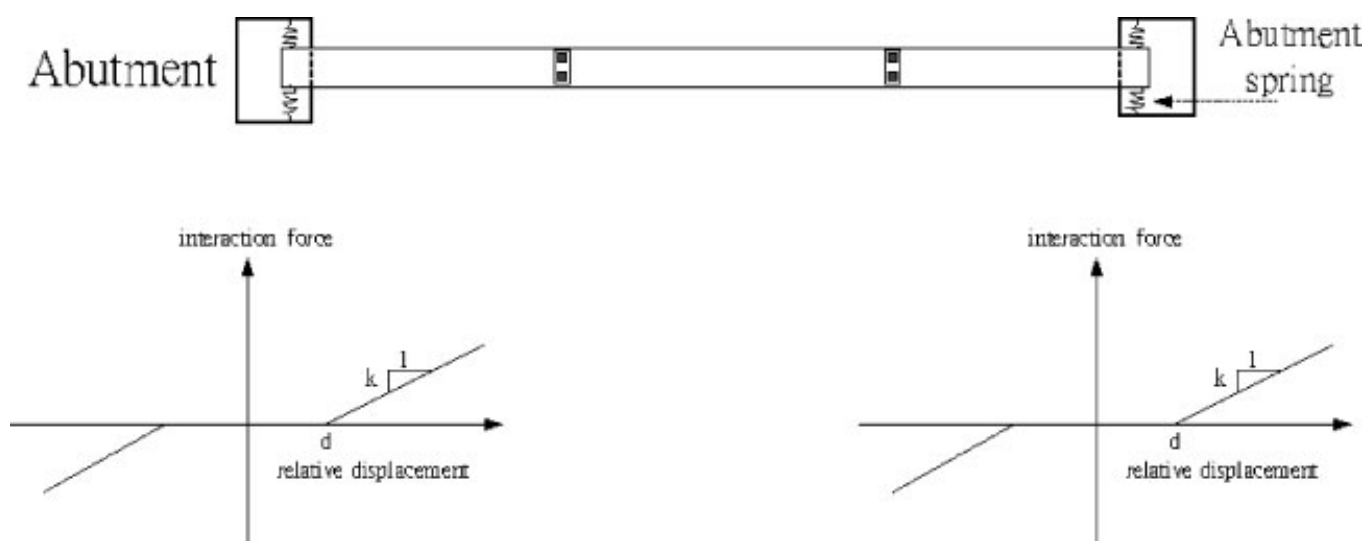

Figure 16. 'Slip-lock' model proposed between the abutments and the deck.

In order to prove the above viewpoints quantitatively, a 'slip-lock' model for the abutment boundary is proposed, as shown in Figure 16. The model is composed of two lock-zones and one slip-zone. The configuration of the three zones is a function of the displacement parameter $d$ and the stiffness parameter $k$. If $d$ tends towards infinity, then the model becomes a 'free end' mechanism. If $k$ tends towards infinity and $d$ approaches zero, then the model becomes a 'hinge end' mechanism. Thus the proposed model with suitable parameters may satisfy the above-mentioned three viewpoints simultaneously. Besides the model and parameters, one more damping coefficient for the deck itself is needed. It is assumed that the damping matrix is directly proportional to the stiffness matrix, i.e.,

$$
[C]=\beta[K]
$$



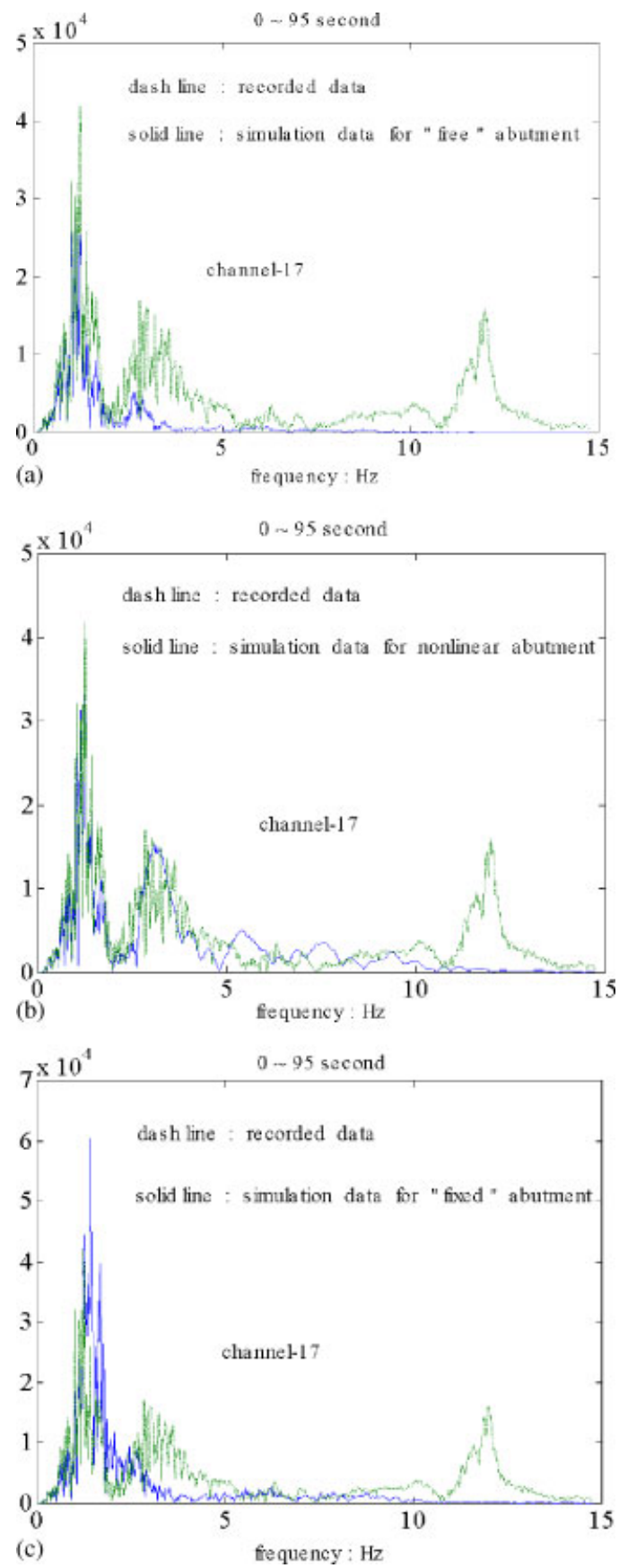

Figure 17. Fourier spectrum comparisons for three models with $\beta=0.0075$ second; (a) free end, (b) slip-lock model, $k=12500 \mathrm{kN} / \mathrm{m}, d=0.01 \mathrm{~m}$, and (c) hinge end. 


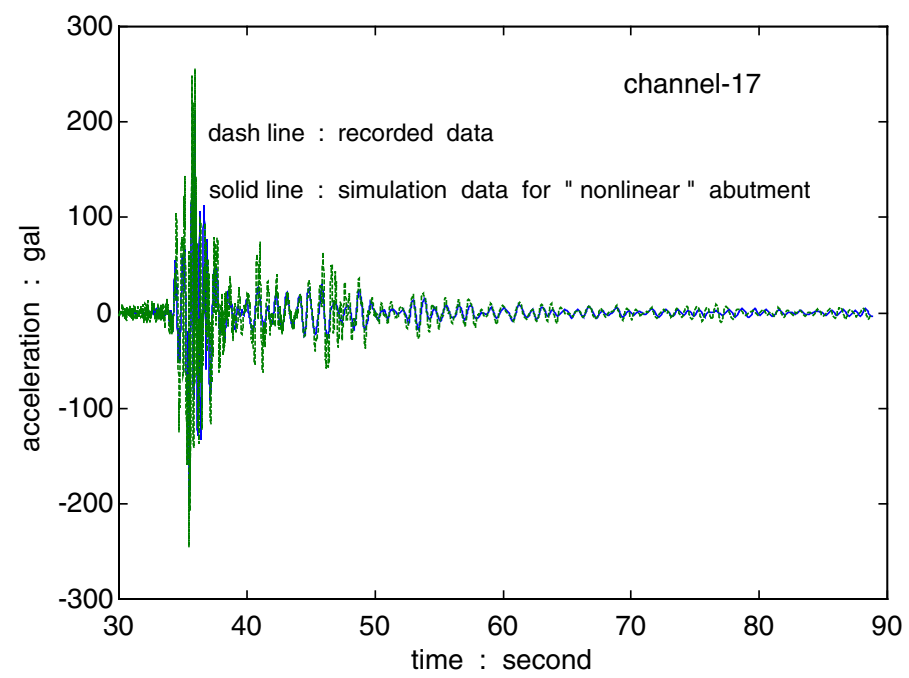

Figure 18. Comparison between the reconstruction response using 'slip-lock model' and recorded data at Channel 17.

There are in total three undetermined parameters $(d, k$ and $\beta)$ to be identified from the response data.

A non-linear dynamic model was developed to identify the above parameters. The average time history of Channel 7 and Channel 10 from the pier foundation is used as the input at the two piers and two abutments. On the other hand, the Fourier spectrum of Channel 17 (all 95-seconds data) is used as the target output to search the optimal parameters. With the three types of boundary condition, Figure 17 shows Fourier spectrum comparisons. It is found that the 'slip-lock' model is the best one to fit the first two dominant frequencies from the data of Channel 17; however, the 'free end' and 'hinge end' mechanisms are incompatible with those frequencies.

From the above study it is concluded that the 'slip-lock' model can fit the first two dominant frequencies of the seismic data, but it mismatches the third vibration frequency. One possible reason is that $\beta=0.0075$ second is too large for the deck around the frequency $12.0 \mathrm{~Hz}$. The frequency dependent $\beta$-value should be implemented to minimize the error in the high-frequency range. For Channel 17, Figure 18 shows the comparison between the seismic response data and the predicted value of the 'slip-lock' model.

\section{CONCLUSIONS}

This paper analyzes the earthquake response of a seismic isolation bridge (Bai-Ho Bridge). From the analysis, the following conclusions are drawn:

1. In this study, both the system realization method and EMD+HHT technique were applied to identify the global behavior of the recorded data. The dominant frequency and damping 
Table III. Dominant frequencies for three different types of vibration (at the center of mid-span).

\begin{tabular}{|c|c|c|c|}
\hline \multicolumn{4}{|c|}{ Ambient vibration test } \\
\hline & Vertical & Transverse & Longitudinal \\
\hline Test no. 1 & $1.8^{*} 3.23 .8$ & $2.4^{\#} 4.2$ & 3.2 \\
\hline Test no. 2 & $1.8^{*} 3.13 .8$ & $2.2^{\#} 2.6$ & 3.1 \\
\hline Test no. 3 & $1.8^{*} 3.23 .8$ & $2.2^{\#} 4.2$ & 3.2 \\
\hline \multicolumn{4}{|c|}{ One moving truck test } \\
\hline Test no. 1 & $1.8^{*} 2.34 .3$ & $2.2^{\#} 2.64 .2$ & 1.32 .83 .4 \\
\hline Test no. 2 & $1.8^{*} 2.53 .8$ & $2.3^{\#} 3.35 .5$ & 1.42 .63 .4 \\
\hline Test no. 3 & $1.8^{*} 2.34 .3$ & $2.2^{\#} 2.6$ & $1.5 \quad 3.5$ \\
\hline \multicolumn{3}{|c|}{ 180-gal scale earthquake } & Longitudinal \\
\hline $1999-10-22$ & $1.8^{*} 4.510 .1$ & 1.33 .112 .1 & 1.112 .0 \\
\hline
\end{tabular}

ratio in the longitudinal direction are identified. The phenomenon of abnormal signals from the abutment are described and figured out by the time-frequency spectrum.

2. In the study of local structures, the mechanism of the LRB isolator is imitated by using the Ramber-Osgood model plus a dashpot model. Not only the model parameters but also the axial stiffness of the deck were identified.

3. Three models, related to the boundary condition at the juncture of the deck and abutment, are selected to examine the transverse vibration of the bridge: the 'free end', 'hinge end' and 'slip-lock' models. The 'hinge end' mechanism, commonly used in design practice, is not appropriate in this study. The proposed 'slip-lock' model appears better than the 'hinge end' model. In the design process, it is better to take the gap and the stiffness of abutments into consideration in the design model.

4. In addition to the seismic response data, a field experiment on the Bai-Ho Bridge was also conducted in order to collect more information on the vibration characteristics of the bridge. Two field tests are included: one is the 'ambient vibration test' and the other is the 'dynamic test by a moving truck'. Table III lists the characteristic frequencies of the field experiment together with those of the 22-10-1999 earthquake at the center of mid-span. From Table III, it is found that the characteristic frequencies are dependent on the intensity and the action-way of disturbances. The reason for the difference in identified vibration frequencies is the non-linearity of the seismic isolator and the effect of the abutment boundary condition in different excitation levels.

\section{ACKNOWLEDGEMENTS}

The authors wish to express thanks to the Central Weather Bureau (CWB) for providing the seismic data for the bridge. The research is sponsored by the National Science Council through Grant no. NSC90-2711-3-319-200-16. The financial support is gratefully acknowledged. 


\section{REFERENCES}

1. Douglas BM, Reid WH. Dynamic tests and system identification of bridges. Journal of Structural Engineering (ASCE) 1982; 108:2295-2312.

2. Werner SD, Beck JL, Levine MB. Seismic response evaluation of Meloland Road Overpass using 1979 Imperial Valley earthquake records. Earthquake Engineering and Structural Dynamics 1987; 15:249-274.

3. Abdel-Ghaffar AM, Scanlan RH. Ambient vibration studies of Golden Gate Bridge: I. Suspended structure. Journal of the Engineering Mechanics Division (ASCE) 1985; 111:463-482.

4. Hoshiya M, Maruyama O. Identification of running load and beam system. Journal of the Engineering Mechanics Division (ASCE) 1987; 113:813-824.

5. Hogue TD, Aktan AE, Hoyos A. Localized identification of constructed facilities. Journal of Structural Engineering (ASCE) 1991; 117:128-148.

6. Loh CH, Lee ZK. Seismic monitoring of a bridge: Assessing dynamic characteristics from both weak and strong ground excitations. Earthquake Engineering and Structural Dynamics 1997; 26:269-288.

7. Loh CH, Lee ZK. System identification of vehicle-bridge interaction. Sino-Canada Bridge Conference, Taipei, March 1998, pp. 174-185.

8. Huang NE, Long SR, Shen Z. The mechanism for frequency downshift in nonlinear wave evolution. Advance Applied Mechanics 1996; 32:59-111.

9. Ljung L, Sörenström T. Theory and Practice of Recursive Identification. MIT Press: Cambridge, MA, 1983.

10. Loh CH. Development of bridge monitoring program in Taiwan. Proceedings of IABSE Conference on CableSupported Bridge, Seoul, Korea, June 2001. 